\title{
Does suppressive antiviral therapy for herpes simplex virus prevent transmission in an HIV-positive population? A systematic review
}

\author{
Smith $\mathrm{CR}^{1,2 *}$, Pogany $\mathrm{L}^{1}$, Auguste $\mathrm{U}^{1}$, Steben $\mathrm{M}^{3}$, Lau TTY ${ }^{4}$
}

\section{Abstract}

Background: Among individuals with genital herpes simplex virus (HSV), co-infection with human immunodeficiency virus (HIV) hs been shown to increase the frequency and severity of HSV symptoms, HSV shedding, and risk of HSV transmission.

Objective: To assess whether suppressive antivirual therapy for genital HSV in an HIV-positive populatation prevents HSV transmission to a susceptible partner.

Methods: A systematic search of the literature was conducted using MEDLINE and EMBASE databases to identify randomized controlled trials published between January 2005 and June 2015. Inclusion criteria were trials written in English or French utilizing suppressive antiviral therapies for HSV. Studies had to report on outcomes related to HSV transmission from HIV-positive populations. Surrogate markers of HSV transmission risk, such as HSV detection and viral load, were also included. Articles underwent a risk of bias assessment, and those with low risk of bias underwent data extraction to complete a narrative synthesis.

Results: This review identified thirteen papers. Only one study directly measured transmission of HSV. The overall transmission rate was $<10 \%$, and suppressive antiviral therapy had no significant protective effect ( $9 \%$ transmission rate in the acyclovir group vs. $6 \%$ in the placebo group; hazard ratio [HR]: $1.35,95 \% \mathrm{Cl}: 0.83-2.20$ ). The remaining 12 papers addressed surrogate markers of transmission risk: HSV detection and viral load. Suppressive acyclovir appears to be effective in reducing HSV detection among HIV-positive populations, but it does not appear to reduce viral load. Suppressive valacyclovir may be effective in reducing HSV detection and viral load among HIV-positive patients who are antiretroviral therapy (ART)-naïve, but its effect appears to be nullified among those concurrently on ART.

Conclusion: Based on current evidence, suppressive antiviral therapy may reduce HSV detection and viral load, but its impact on HSV transmission is unclear. Clinicians should caution HIV-positive patients with HSV that suppressive therapy may not reduce risk of HSV transmission to susceptible partners.

\section{Affiliations}

${ }^{1}$ Centre for Communicable Diseases and Infection Control, Infectious Disease Prevention and Control Branch, Public Health Agency of Canada, Ottawa, ON

${ }^{2}$ Dalla Lana School of Public Health, University of Toronto, Toronto, ON

${ }^{3}$ STI Unit, Institut national de santé publique du Québec, Montréal, QC

${ }^{4}$ Pharmaceutical Sciences, Vancouver General Hospital, Vancouver Coastal Health, and Faculty of Pharmaceutical Sciences, University of British Columbia, Vancouver, BC

*Correspondence: courtneyrady. smith@mail.utoronto.ca

Suggested citation: Smith CR, Pogany L, Auguste U, Steben M, Lau TTY. Does suppressive antiviral therapy for herpes simplex virus prevent transmission in an HIV-positive population? A systematic review. Can Comm Dis Rep 2016;42-2:37-44. https://doi.org/10.14745/ccdr.v42i02a03

\section{Introduction}

Approximately $14 \%$ of Canadian adults tested positive for genital herpes simplex virus (HSV) type 2 in 2009 (1). HSV is particularly widespread among people with human immunodeficiency virus type 1 (HIV), affecting $50 \%$ to $90 \%$ of the HIV-positive population (2).

Genital HSV reactivation has been shown to increase HIV viral load, enhancing risk of HIV transmission and HIV disease progression $(3,4)$. In turn, HIV has been shown to increase the frequency and severity of HSV symptoms, HSV shedding, and risk of HSV transmission $(5,6)$. Given this interaction between HSV and HIV, prevention of HSV transmission among HIV-positive populations is a significant public health concern. Minimizing rates of HSV co-infection among HIV-positive individuals could prevent increases in HIV viral load, HIV transmission, and HIV progression that characterize HSV and HIV co-infection. Preventing HSV transmission from HIV-positive partners to HIV-negative partners is also important to public health as HSV infection has been estimated to increase the risk of HIV acquisition three-fold (7). 
Suppressive therapy with acyclovir, famciclovir, or valacyclovir is routinely used in populations co-infected with HSV and HIV. These agents have been shown to reduce HSV detection and viral load, which have been linked to a reduced risk of HSV transmission in immunocompetent populations $(8,9)$. Of interest is whether these agents also reduce risk of HSV transmission in HIV-positive populations. The most recent reviews, published in $2007(10,11)$ but neither were able to find sufficient literature to evaluate HSV transmission in HIVpositive populations.

The objective of this systematic review was to assess randomized controlled trials in the HSV and HIV co-infected population, focusing on the impact of suppressive HSV antiviral therapies on HSV transmission, HSV detection, and HSV viral load.

\section{Methods}

\section{Search strategy}

A systematic search of MEDLINE and EMBASE databases was conducted to find articles on randomized controlled trials published within the last 10 years; previous reviews included searches for literature published prior to this. The search strategy used MeSH terms for "treatment outcome" and "herpes simplex virus," along with relevant keywords (see text box).

\begin{tabular}{|c|c|}
\hline$\#$ & Search strategy \\
\hline 1 & exp treatment outcome/ \\
\hline 2 & drug efficacy/ or drug effect/ \\
\hline 3 & $\left(\right.$ effic ${ }^{\star}$ or outcome*).mp. \\
\hline 4 & 1 or 2 or 3 \\
\hline 5 & $\begin{array}{l}\text { exp *herpes simplex/ or (herpes or HSV1 or HSV2 or HSV-1 or } \\
\text { HSV-2 or HSV).tw. }\end{array}$ \\
\hline 6 & 4 and 5 \\
\hline 7 & limit 6 to (randomized controlled trial and last 10 years) \\
\hline
\end{tabular}

\section{Eligibility criteria}

Articles were eligible if they were written in English or French and had been published between January 2005 and June 2015 in peer-reviewed journals. Studies needed to report on genital HSV in HIV-positive adults (18 years or older) and describe a pharmacological intervention using a randomized controlled trial design. Articles were only included if the pharmacological comparison evaluated a suppressive treatment with at least one of the three most commonly used anti-HSV oral agents, acyclovir, famciclovir and/or valacyclovir. All eligible articles had to report on HSV transmission, detection, and/or viral load as outcomes. Studies that assessed topical treatments (e.g. gels or creams) were excluded due to their lack of availability outside the scope of clinical trials and the limited efficacy of the treatment. Studies in pregnancy and those that examined episodic treatments were outside the scope of the research question.

\section{Study selection}

Titles were screened and excluded if they were not related to $\mathrm{HSV}$ or HIV. Articles meeting this basic screening criterion were obtained for full-text review and assessed independently by two authors (CRS, UA) based on the eligibility criteria described above; a review resolved any discrepancies. References from relevant articles were screened and retrieved where appropriate. A formal quality assessment of each article meeting all the eligibility criteria was then performed independently by two authors (CRS, UA), using the Cochrane Collaboration's tool for assessing risk of bias (12). Studies with a high risk of bias were excluded.

\section{Data extraction}

Data were summarized by one author (CRS) in Microsoft Excel and then reviewed for accuracy and completeness by another (UA). Relevant data for each article included the patient population, country of study, pharmacological regimens, follow-up period, antiretroviral therapy (ART) status, CD4 cell count, number of transmissions of HSV (primary outcome), HSV detection and viral load (surrogate markers of transmission risk), effect measures, and adverse events.

The purpose of this review was to provide a narrative synthesis of the literature, so funnel plots and assessment of heterogeneity were not completed. A review protocol was not published for this review.

\section{Results}

A total of 492 papers were identified, 485 from databases and 7 through reference searches of relevant articles. Of these, 315 were unique. After screening and eligibility assessment, 14 studies remained to be assessed for risk of bias. One paper was excluded due to a high risk of bias (13) resulting in 13 papers being included in the systematic review (Appendix 1).

\section{Study characteristics}

The characteristics of the 13 studies are summarized in Table 1. Only one of the 13 studies directly measured HSV transmission (14). The remaining 12 papers focused on surrogate markers of transmission risk, that is, HSV viral detection and/or viral load. Of these, 7 studies addressed both HSV detection and genital HSV viral load $(15,17,19,21-24)$, whereas 5 reported on HSV detection alone $(16,17,19,23,24)$. All the studies utilized polymerase chain reaction (PCR) assays for both HSV detection and viral load.

The 13 clinical trials included 2,367 HSV and HIV co-infected participants. The majority of trials were conducted on populations in African countries ( $n=7)$, and two were in Peru, two in the United States, and one in Thailand. One trial used a sample that spanned three continents. The majority of studies involved only female participants $(n=9)$, one used only males, and three included both sexes. Follow-up periods varied from 1 to 24 months. Eight studies included only participants who were 
Table 1: Characteristics of suppressive antiviral therapy for herpes simplex virus in an HIV-positive population

\begin{tabular}{|c|c|c|c|c|c|c|}
\hline $\begin{array}{l}\text { Authors } \\
\text { Year }\end{array}$ & Number $^{1}$ & $\begin{array}{l}\text { Country of } \\
\text { study }\end{array}$ & $\begin{array}{l}\text { Pharmacological } \\
\text { regimen }\end{array}$ & $\begin{array}{l}\text { Follow-up } \\
\text { months }\end{array}$ & HIV treatment & Outcome measure \\
\hline $\begin{array}{l}\text { Mujugira et al. (14) } \\
2013\end{array}$ & $\begin{array}{l}911 \text { males and } \\
\text { females }\end{array}$ & $\begin{array}{l}7 \text { (eastern and } \\
\text { southern African } \\
\text { countries) }\end{array}$ & $\begin{array}{l}\text { Acyclovir } \\
\text { ( } 400 \mathrm{mg} \text { bid) versus } \\
\text { placebo }\end{array}$ & 24 & $\begin{array}{l}\text { Not receiving } \\
\text { Antiretroviral therapy } \\
\text { (ART) }\end{array}$ & HSV-2 transmission \\
\hline $\begin{array}{l}\text { Baeten et al. (15) } \\
2008\end{array}$ & 20 females & Peru & $\begin{array}{l}\text { Valacyclovir } \\
\text { ( } 500 \mathrm{mg} \text { bid) versus } \\
\text { placebo }\end{array}$ & 2 & Not receiving ART & $\begin{array}{l}\text { Genital HSV-2 detection } \\
\text { Genital HSV-2 viral load } \\
\text { Adverse events }\end{array}$ \\
\hline $\begin{array}{l}\text { Cowan et al. (16) } \\
2008\end{array}$ & 125 females & Zimbabwe & $\begin{array}{l}\text { Acyclovir } \\
\text { ( } 400 \mathrm{mg} \text { bid) versus } \\
\text { placebo }\end{array}$ & 3 & $\begin{array}{l}\text { Not explicitly stated } \\
\text { (however, ART was } \\
\text { rarely available at the } \\
\text { time of the study) }\end{array}$ & Genital HSV-2 detection \\
\hline $\begin{array}{l}\text { Delany et al. (17) } \\
2009\end{array}$ & 300 females & South Africa & $\begin{array}{l}\text { Acyclovir } \\
\text { ( } 400 \mathrm{mg} \text { bid) versus } \\
\text { placebo }\end{array}$ & 3 & Not receiving ART & $\begin{array}{l}\text { Genital HSV-2 detection } \\
\text { Genital HSV-2 viral load } \\
\text { Adverse events }\end{array}$ \\
\hline $\begin{array}{l}\text { Dunne et al. (18) } \\
2008\end{array}$ & 67 females & Thailand & $\begin{array}{l}\text { Acyclovir } \\
\text { ( } 800 \mathrm{mg} \text { bid) versus } \\
\text { placebo }\end{array}$ & 1 & Not receiving ART & Genital HSV-2 detection \\
\hline Kim at al. (19) 2010 & $\begin{array}{l}76 \text { males and } \\
\text { females }\end{array}$ & $\begin{array}{l}\text { South Africa, } \\
\text { Zimbabwe, Zambia, } \\
\text { Peru, United States }\end{array}$ & $\begin{array}{l}\text { Acyclovir } \\
\text { ( } 400 \mathrm{mg} \text { bid) versus } \\
\text { placebo }\end{array}$ & 6 & Not receiving ART & $\begin{array}{l}\text { Genital HSV-2 detection } \\
\text { Genital HSV-2 viral load }\end{array}$ \\
\hline $\begin{array}{l}\text { Nagot et al. (20) } \\
2007\end{array}$ & 140 females & Burkina Faso & $\begin{array}{l}\text { Valacyclovir } \\
\text { ( } 500 \mathrm{mg} \text { bid) versus } \\
\text { placebo }\end{array}$ & 3 & Not receiving ART & $\begin{array}{l}\text { Genital HSV-2 detection } \\
\text { Adverse events }\end{array}$ \\
\hline $\begin{array}{l}\text { Ouedraogo et al. } \\
\text { (21) } 2006\end{array}$ & 60 females & Burkina Faso & $\begin{array}{l}\text { Valacyclovir } \\
\text { ( } 500 \mathrm{mg} \text { bid) versus } \\
\text { placebo }\end{array}$ & 3 & $\begin{array}{l}\text { All patients receiving } \\
\text { highly active } \\
\text { antiretroviral therapy } \\
\text { (HAART) }\end{array}$ & $\begin{array}{l}\text { Genital HSV-2 detection } \\
\text { Genital HSV-2 viral load }\end{array}$ \\
\hline Perti et al. (22) 2013 & $\begin{array}{l}34 \text { males and } \\
\text { females }\end{array}$ & United States & $\begin{array}{l}\text { Acyclovir } \\
\text { ( } 400 \mathrm{mg} \text { bid) versus } \\
\text { valacyclovir } \\
(1,000 \mathrm{mg} \text { bid })\end{array}$ & 3 & Not receiving ART & $\begin{array}{l}\text { Genital HSV-2 detection } \\
\text { Genital HSV-2 viral load } \\
\text { Adverse events }\end{array}$ \\
\hline $\begin{array}{l}\text { Tanton et al. (23) } \\
2010\end{array}$ & 484 females & Tanzania & $\begin{array}{l}\text { Acyclovir } \\
\text { ( } 400 \mathrm{mg} \text { bid) versus } \\
\text { placebo }\end{array}$ & 24 & $\begin{array}{l}\text { Not explicitly stated } \\
\text { (Free ART became } \\
\text { available in regional } \\
\text { and district hospitals } \\
\text { in the study area } \\
\text { during the trial) }\end{array}$ & $\begin{array}{l}\text { Genital HSV-2 detection } \\
\text { Genital HSV-2 viral load } \\
\text { Adverse events }\end{array}$ \\
\hline $\begin{array}{l}\text { Tobian et al. (24) } \\
2013\end{array}$ & $96^{1}$ females & Uganda & $\begin{array}{l}\text { Acyclovir } \\
\text { ( } 400 \mathrm{mg} \text { bid) versus } \\
\text { placebo }\end{array}$ & 24 & $\begin{array}{l}\text { All patients receiving } \\
\text { ART }\end{array}$ & $\begin{array}{l}\text { Genital HSV-2 detection } \\
\text { Genital HSV-2 viral load }\end{array}$ \\
\hline $\begin{array}{l}\text { Van Wagoner et al. } \\
\text { (25) } 2015\end{array}$ & $34^{2}$ females & United States & $\begin{array}{l}\text { Valacyclovir } \\
(1,000 \mathrm{mg} \text { qd) versus } \\
\text { placebo }\end{array}$ & 6 & $\begin{array}{l}\text { All patients receiving } \\
\text { ART }\end{array}$ & $\begin{array}{l}\text { Genital HSV-2 detection } \\
\text { Adverse events }\end{array}$ \\
\hline $\begin{array}{l}\text { Zuckerman et al. } \\
\text { (26) } 2007\end{array}$ & 20 males & Peru & $\begin{array}{l}\text { Valacyclovir } \\
\text { ( } 500 \mathrm{mg} \text { bid) versus } \\
\text { placebo }\end{array}$ & 2 & Not receiving ART & $\begin{array}{l}\text { Genital HSV-2 detection } \\
\text { Adverse events }\end{array}$ \\
\hline
\end{tabular}

Abbreviations: ART, antiretroviral therapy; HAART, highly active antiretroviral therapy; HIV, human immunodeficiency virus; HSV-1, herpes simplex virus type-1; HSV-2, herpes simplex virus type-2

1440 subjects were enrolled, but only 96 were assessed for genital HSV-2 detection/genital HSV-2 viral load

2101 subjects were enrolled, but only 34 were assessed for genital HSV-2 detection 
ART-naïve, three included only individuals receiving ART, and two did not explicitly state ART status. Most trials either compared suppressive acyclovir (400 mg bid or $800 \mathrm{mg}$ bid) to placebo $(n=7)$ or suppressive valacyclovir $(500 \mathrm{mg}$ bid or $1,000 \mathrm{mg} \mathrm{qd}$ ) to placebo $(\mathrm{n}=5)$. One study compared suppressive acyclovir (400 mg bid) to high-dose suppressive valacyclovir (1,000 mg bid). None of the studies used famciclovir. All clinical trials were evaluated to be of low risk of bias.

\section{HSV-2 transmission}

HSV-2 transmission was only directly measured in one study, a well-designed trial where 911 sero-discordant heterosexual couples were followed for 24 months (14). Infected partners were HSV positive and HIV positive (not on ART) and were randomized to either suppressive treatment (acyclovir $400 \mathrm{mg}$ bid) or placebo. Susceptible partners were HSV negative and HIV negative. In this study, suppressive acyclovir did not reduce transmission of HSV when compared with placebo. Transmission occurred in 9\% (40/458) of the treatment group and in $6 \%(28 / 453)$ of the placebo group (hazard ratio [HR]: 1.35, 95\% confidence interval [CI]: 0.83-2.20). HSV transmission was two times higher from males to females than from females to males.

\section{HSV-2 detection}

Twelve studies reported on genital HSV detection. These studies utilized PCR and reported the percentage of participants, visits, or swabs/samples that were positive for HSV.

\section{Acyclovir versus placebo}

Six clinical trials that reported on HSV detection compared suppressive acyclovir to placebo (Table 2). Five of the six trials used a standard dose of acyclovir (400 mg bid) $(16,17,19,23,24)$, whereas one had a higher dose of $800 \mathrm{mg}$ bid (18). Overall, four of the six studies found a statistically significant effect of suppressive acyclovir treatment on HSV detection; two studies with ART-naïve patients $(17,18)$ one with patients who initiated ART at study commencement (24), and one where ART status $\mathrm{mg}$ bid dose appeared to have greater efficacy than $400 \mathrm{mg}$ bid doses. Two of the six trials (both using a $400 \mathrm{mg}$ bid dose) reported null findings-one with ART-naïve participants (19), and one where ART status was not stated (23). Only one of the acyclovir studies included both males and females, but this study did not report results by sex.

\section{Valacyclovir versus placebo}

Five clinical trials that reported on HSV detection compared suppressive valacyclovir to placebo (Table 3). Four studies used a $500 \mathrm{mg}$ bid dose $(15,20,21,26)$, whereas one used $1,000 \mathrm{mg}$ qd (25). Three studies (all using a $500 \mathrm{mg}$ bid dose) reported a statistically significant protective effect of suppressive

Table 2: Summarized results for suppressive acyclovir compared to placebo

\begin{tabular}{|c|c|c|c|c|c|c|c|c|}
\hline \multirow[b]{2}{*}{ Study } & \multirow{2}{*}{$\begin{array}{l}\text { Acyclovir } \\
\text { dose }\end{array}$} & \multirow{2}{*}{$\begin{array}{l}\text { Treatment for } \\
\text { HIV }\end{array}$} & \multicolumn{3}{|c|}{$\begin{array}{c}\text { Genital herpes simplex virus (HSV) } \\
\text { detection }\end{array}$} & \multicolumn{3}{|c|}{ Genital HSV viral load ${ }^{1}$} \\
\hline & & & $\begin{array}{l}\text { Proportion } \\
\text { treatment } \\
\text { group }\end{array}$ & $\begin{array}{c}\text { Proportion } \\
\text { placebo } \\
\text { group }\end{array}$ & $\begin{array}{c}\text { Estimate of } \\
\text { effect }\end{array}$ & $\begin{array}{l}\text { Treatment } \\
\text { group }\end{array}$ & $\begin{array}{l}\text { Placebo } \\
\text { group }\end{array}$ & $P$ value \\
\hline $\begin{array}{l}\text { Cowan et al., } \\
2008(16)\end{array}$ & $400 \mathrm{mg}$ bid & $\begin{array}{l}\text { Not explicitly } \\
\text { stated }\end{array}$ & $10 \%$ of visits & $23 \%$ of visits & $\begin{array}{l}\mathrm{OR}=0.24 \\
(95 \% \text { Cl: } 0.12- \\
0.48)\end{array}$ & & & \\
\hline $\begin{array}{l}\text { Delany et al., } \\
2009(17)\end{array}$ & $400 \mathrm{mg}$ bid & $\begin{array}{l}\text { Not receiving } \\
\text { ART }\end{array}$ & $\begin{array}{l}33 \% \text { of } \\
\text { patients }\end{array}$ & $\begin{array}{l}54 \% \text { of } \\
\text { patients }\end{array}$ & $\begin{array}{l}\mathrm{RR}=0.61(95 \% \\
\mathrm{Cl}: 0.46-0.80)\end{array}$ & Mean $=3.38$ & $\begin{array}{l}\text { Mean = } \\
3.81\end{array}$ & $p=0.13$ \\
\hline $\begin{array}{l}\text { Kim et al., } \\
2010 \text { (19) }\end{array}$ & $400 \mathrm{mg}$ bid & $\begin{array}{l}\text { Not receiving } \\
\text { ART }\end{array}$ & $\begin{array}{l}19.4 \% \text { of } \\
\text { patients }\end{array}$ & $\begin{array}{l}22.5 \% \text { of } \\
\text { patients }\end{array}$ & $\begin{array}{l}\text { Not stated (but } \\
p=0.07)\end{array}$ & Median $=6.50$ & $\begin{array}{l}\text { Median = } \\
6.90\end{array}$ & $p=0.91$ \\
\hline $\begin{array}{l}\text { Tanton et al., } \\
2010(23)\end{array}$ & $400 \mathrm{mg}$ bid & $\begin{array}{l}\text { Not explicitly } \\
\text { stated }\end{array}$ & $10.9 \%$ of visits & $11.8 \%$ of visits & $\begin{array}{l}\mathrm{OR}=0.90 \\
(95 \% \mathrm{Cl}: 0.60- \\
1.36)\end{array}$ & Mean $=4.16$ & $\begin{array}{l}\text { Mean = } \\
4.07\end{array}$ & $p=0.73$ \\
\hline $\begin{array}{l}\text { Tobian et al., } \\
2013 \text { (24) }\end{array}$ & $400 \mathrm{mg}$ bid & $\begin{array}{l}\text { All patients } \\
\text { receiving ART }\end{array}$ & $1.4 \%$ of visits & $10.2 \%$ of visits & $\begin{array}{l}\mathrm{OR}=0.13 \\
(95 \% \mathrm{Cl}: 0.04- \\
0.41)\end{array}$ & Median $=3.52$ & $\begin{array}{l}\text { Median = } \\
3.57\end{array}$ & $p=0.82$ \\
\hline $\begin{array}{l}\text { Dunne et al., } \\
2008 \text { (18) }\end{array}$ & $800 \mathrm{mg}$ bid & $\begin{array}{l}\text { Not receiving } \\
\text { ART }\end{array}$ & $\begin{array}{l}1.6 \% \text { of } \\
\text { patients }\end{array}$ & $\begin{array}{l}42.4 \% \text { of } \\
\text { patients }\end{array}$ & $\begin{array}{l}\mathrm{RR}=0.00(95 \% \\
\mathrm{Cl}: .006-0.33)\end{array}$ & & & \\
\hline
\end{tabular}

Abbreviations: ART, antiretroviral therapy; $\mathrm{Cl}$, confidence interval; HAART, highly active antiretroviral therapy; OR, odds ratio; RR, relative ratio

1 Log10 copies/mL among those with detectable HSV-2 DNA 
Table 3: Summarized results for suppressive valacyclovir compared to placebo

\begin{tabular}{|c|c|c|c|c|c|c|c|c|}
\hline \multirow[b]{2}{*}{ Study } & \multirow[b]{2}{*}{$\begin{array}{l}\text { Valacyclovir } \\
\text { dose }\end{array}$} & \multirow[b]{2}{*}{$\begin{array}{l}\text { Treatment for } \\
\text { HIV }\end{array}$} & \multicolumn{3}{|c|}{$\begin{array}{c}\text { Genital herpes simplex virus (HSV) } \\
\text { detection }\end{array}$} & \multicolumn{3}{|c|}{ Genital HSV viral load ${ }^{1}$} \\
\hline & & & $\begin{array}{l}\text { Proportion } \\
\text { treatment } \\
\text { group }\end{array}$ & $\begin{array}{c}\text { Proportion } \\
\text { placebo } \\
\text { group }\end{array}$ & $\begin{array}{c}\text { Estimate of } \\
\text { effect }\end{array}$ & $\begin{array}{l}\text { Treatment } \\
\text { group }\end{array}$ & $\begin{array}{l}\text { Placebo } \\
\text { group }\end{array}$ & $\begin{array}{c}P \\
\text { value }\end{array}$ \\
\hline $\begin{array}{l}\text { Baeten et al., } \\
2008(15)\end{array}$ & $500 \mathrm{mg} \mathrm{bid}$ & $\begin{array}{l}\text { Not receiving } \\
\text { ART }\end{array}$ & $\begin{array}{l}3.7 \% \text { of } \\
\text { samples }\end{array}$ & $\begin{array}{l}22.1 \% \text { of } \\
\text { samples }\end{array}$ & $\begin{array}{l}\text { OR }=0.13 \\
(95 \% \text { Cl: } 0.07- \\
0.24)\end{array}$ & & Mean $=4.80$ & $\begin{array}{l}p= \\
0.002\end{array}$ \\
\hline $\begin{array}{l}\text { Nagot et al., } \\
2007(20)\end{array}$ & $500 \mathrm{mg}$ bid & $\begin{array}{l}\text { Not receiving } \\
\text { ART }\end{array}$ & $4.1 \%$ of visits & $17.9 \%$ of visits & $\begin{array}{l}\mathrm{RR}=0.29 \\
(95 \% \mathrm{Cl}: 0.14- \\
0.58)\end{array}$ & & & \\
\hline $\begin{array}{l}\text { Ouedraogo et } \\
\text { al., } 2006(21)\end{array}$ & $500 \mathrm{mg} \mathrm{bid}$ & $\begin{array}{l}\text { All patients } \\
\text { receiving } \\
\text { highly active } \\
\text { antiretroviral } \\
\text { therapy (HAART) }\end{array}$ & $6.6 \%$ of visits & $9.8 \%$ of visits & $\begin{array}{l}\mathrm{OR}=0.37 \\
(95 \% \mathrm{Cl}: 0.13- \\
1.05)\end{array}$ & & $P$ value & $\begin{array}{l}p= \\
0.12\end{array}$ \\
\hline $\begin{array}{l}\text { Van Wagoner } \\
\text { et al., } 2015 \\
(25)\end{array}$ & $1,000 \mathrm{mg}$ qd & $\begin{array}{l}\text { All patients } \\
\text { receiving ART }\end{array}$ & $\begin{array}{l}3.8 \% \text { of } \\
\text { patients }\end{array}$ & $\begin{array}{l}12.5 \% \text { of } \\
\text { patients }\end{array}$ & Not stated & & & \\
\hline $\begin{array}{l}\text { Zuckerman et } \\
\text { al., } 2007 \text { (26) }\end{array}$ & $500 \mathrm{mg}$ bid & $\begin{array}{l}\text { Not receiving } \\
\text { ART }\end{array}$ & $4 \%$ of samples & $\begin{array}{l}29 \% \text { of } \\
\text { samples }\end{array}$ & $\begin{array}{l}\text { Not stated } \\
\text { (but } p<0.001 \text { ) }\end{array}$ & & & \\
\hline
\end{tabular}

Abbreviations: ART, antiretroviral therapy; $\mathrm{Cl}$, confidence interval; HAART, highly active antiretroviral therapy; OR, odds ratio; RR, relative ratio

1 Log10 copies/mL among those with detectable HSV-2 DNA

valacyclovir on HSV detection $(15,20,26)$, whereas one (also using a $500 \mathrm{mg}$ bid dose) did not (21). One study (using a $1,000 \mathrm{mg}$ qd dose) did not compare HSV detection between groups because of limited HSV detection in their sample (25). Of the three studies that reported a significant effect, all were in ART-naive patients. The two studies that reported a null finding (or could not statistically compare groups) both had participants concurrently on ART. None of the valacyclovir studies sampled both males and females, and thus any differences based on sex could not be reported.

\section{Acyclovir versus valacyclovir}

One study directly compared suppressive acyclovir (400 mg bid) to a high-dose suppressive valacyclovir group $(1,000 \mathrm{mg}$ bid) in ART-naïve participants (22) (Table 4). There was no significant difference in genital HSV detection between groups. Although this study did include males and females, results were not stratified by gender.

\section{HSV-2 viral load}

Of the 12 studies that reported on genital HSV detection, 7 also reported the HSV viral load. These studies utilized PCR and reported either mean or median values for log10 copies $/ \mathrm{mL}$, with greater values suggestive of a potential increased risk of transmission.

\section{Acyclovir versus placebo}

Four clinical trials that compared suppressive acyclovir (400 mg bid) to placebo reported on genital HSV viral load $(17,19,23,24)$ (Table 2). There were no significant differences in viral load between groups in any of these studies. Two studies had ART-naïve participants $(17,19)$, one had patients concurrently taking ART (24), and one did not state ART status (23). Only one of the acyclovir studies included both males and females, but this study did not report results based on sex.

\section{Valacyclovir versus placebo}

Two studies that compared suppressive valacyclovir (500 mg bid) to placebo reported on genital HSV viral load $(15,21)$ (Table 3 ). One study found valacyclovir significantly reduced viral load in ART-naïve participants (15). The other did not find a significant difference between groups in a population concurrently on highly active ART (21). Neither study sampled both sexes so differences based on sex could not be noted.

\section{Acyclovir versus valacyclovir}

Compared to high-dose suppressive valacyclovir (1,000 mg bid), suppressive acyclovir (400 mg bid) demonstrated no significant difference in viral load among ART-naïve participants (22) (Table 4). Although this study did include males and females, results were not stratified by sex. 
Table 4: Summarized results for suppressive acyclovir compared to suppressive valacyclovir

\begin{tabular}{|c|c|c|c|c|c|c|c|c|}
\hline \multirow[b]{2}{*}{ Study } & \multirow[b]{2}{*}{ Dose } & \multirow[b]{2}{*}{$\begin{array}{l}\text { Treatment for } \\
\text { HIV }\end{array}$} & \multicolumn{3}{|c|}{$\begin{array}{c}\text { Genital herpes simplex virus (HSV) } \\
\text { detection }\end{array}$} & \multicolumn{3}{|c|}{ Genital HSV viral load } \\
\hline & & & $\begin{array}{c}\text { Proportion } \\
\text { Acyclovir } \\
\text { group }\end{array}$ & $\begin{array}{c}\text { Proportion } \\
\text { Valacyclovir } \\
\text { group }\end{array}$ & $\begin{array}{l}\text { Estimate } \\
\text { of effect }\end{array}$ & $\begin{array}{c}\text { Acyclovir } \\
\text { treatment } \\
\text { group }\end{array}$ & $\begin{array}{c}\text { Valacyclovir } \\
\text { treatment } \\
\text { group }\end{array}$ & $\begin{array}{c}P \\
\text { value }\end{array}$ \\
\hline $\begin{array}{l}\text { Perti et al., } \\
\text { 2013(22) }\end{array}$ & $\begin{array}{l}\text { Valacyclovir } \\
\text { group: } \\
\text { 1,000 mg bid } \\
\text { Acyclovir } \\
\text { group: } \\
400 \text { mg bid }\end{array}$ & $\begin{array}{l}\text { Not receiving } \\
\text { ART }\end{array}$ & $8.2 \%$ of days & $7.8 \%$ of days & $\begin{array}{l}\mathrm{RR}=0.95 \\
(95 \% \mathrm{Cl}: \\
0.66-1.37)\end{array}$ & Median $=3.0$ & Median $=3.0$ & $p=0.67$ \\
\hline
\end{tabular}

Abbreviations: ART, antiretroviral therapy; $\mathrm{Cl}$, confidence interval; $\mathrm{RR}$, relative ratio

\section{Adverse events}

\section{Acyclovir versus placebo}

Of the seven clinical trials comparing acyclovir to placebo, only two reported on adverse events $(17,23)$ both trials noted comparable rates in acyclovir and placebo groups. Adverse events included bacterial infections unrelated to treatment, HIV-related events, and malaria.

\section{Valacyclovir versus placebo}

Of the five studies comparing valacyclovir to placebo, four reported on adverse events. Two studies reported no serious adverse events, without going into any further detail $(15,26)$. The remaining two studies had similar rates of adverse events in valacyclovir and placebo groups $(20,25)$. One of these studies summarized frequencies of adverse events, with the most common being headaches $(29 \%$ and $40 \%$, in the valacyclovir group and placebo group, respectively), hypersensitivity reactions (15\% and $21 \%)$, fatigue (15\% and $25 \%)$, and nausea $(16 \%$ and $10 \%)(20)$.

\section{Acyclovir versus valacyclovir}

The study comparing acyclovir to high-dose valacyclovir reported two adverse events related to high-dose valacyclovir (22). One participant developed urticaria and two participants with depression developed exacerbations.

\section{Discussion}

In our systematic review, only one study directly examined HSV transmission from an HIV-positive population and found no significant difference between suppressive acyclovir and placebo. Although this study had a large sample size and a lengthy follow-up period, confirmation of this finding is needed. Most studies included in this review focused on surrogate markers of HSV transmission risk, which included HSV detection and viral load. Overall, suppressive acyclovir appears to be successful in reducing HSV detection among HIV-positive populations, but it does not effectively reduce viral load. Given variations in acyclovir absorption (27), this contrary finding between HSV detection and HSV viral load may not be surprising. The effect of suppressive valacyclovir may be linked to ART status. Although this observation was based on a small sample of studies, it appears that ART may nullify the otherwise significant impact of valacyclovir therapy on HSV detection and viral load. However, this result should be interpreted with caution, as it could be confounded by the fact that individuals eligible for ART may be at a more advanced stage of HIV. In addition, the studies included in this review were not designed to specifically evaluate the impact of ART.

This systematic review provides updated information on a topic important to public health. Although previous reports assessed the impact of suppressive antiviral treatment on HSV and HIV co-infection, they were unable to specifically address HSV transmission in this population. One of the main strengths of our review is that it summarizes recent literature with a low risk of bias. In addition, all studies utilized PCR for HSV detection and viral load, overcoming the limitations of culture, which may vary more widely between studies. Based on our evaluation of the published literature, when prescribing suppressive antiviral treatment for those co-infected with HSV and HIV, clinicians should clearly articulate that treatment may not necessarily reduce risk of HSV transmission to an un-infected partner.

Further work is needed to confirm whether higher doses of acyclovir have a greater impact on viral load, and whether valacyclovir is only effective in ART-naïve populations. In addition, whereas resistance to acyclovir and valacyclovir is extremely low among immunocompetent populations, the resistance rate is approximately $5 \%$ in the HIV-positive population in North America $(27,28)$. Further studies among patients with HSV and HIV are needed to address the impact of the notable level of resistance in this specific population.

Limitations of this review include the potential for publication bias and the exclusion of non-randomized controlled trials. Within the clinical trials, sample sizes, population characteristics, 
types of ART, follow-up periods, and reporting methods varied. Although most studies required participants to have a

CD4 count higher than 200 cells/ $\mu \mathrm{L}$, there was variation within studies. Dosing regimens for antivirals also varied. In addition, biological measures of adherence were not used in any study; thus, reported compliance may have been inaccurate. It is also important to note that all of the studies addressing detection and viral load were powered on HIV-related outcomes, rather than the outcomes of interest in this review. As a result, it is possible that in some cases, sample sizes may have been too

\section{Appendix 1: Flowchart showing selection of} randomized control trials for review

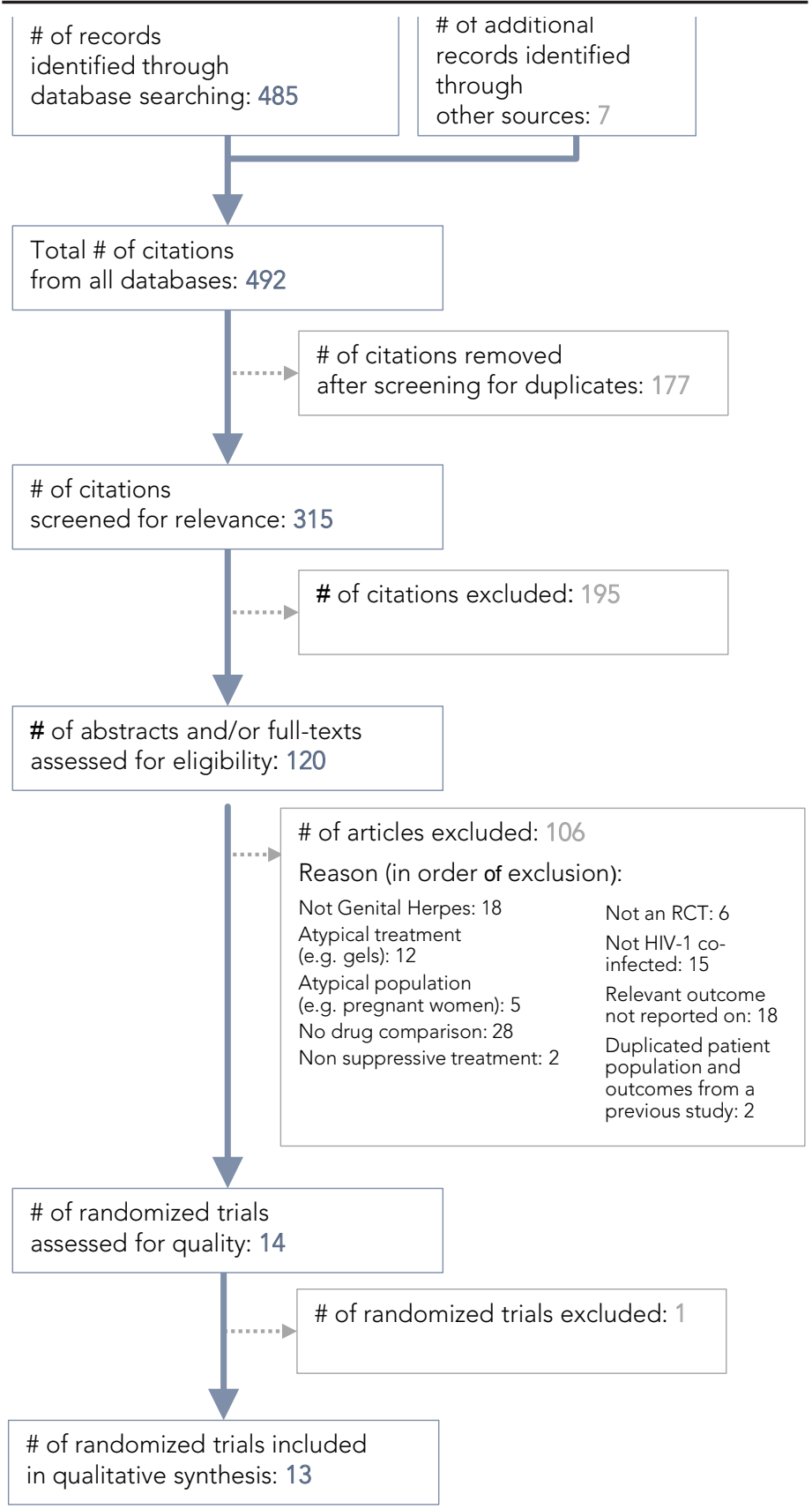

small to detect a difference in HSV detection or viral load. Lastly, the majority of studies were set in developing countries, with heterosexual populations; the generalizability of these results to a Canadian context and to populations of men who have sex with men may be limited.

\section{Conclusion}

Although suppressive antiviral therapies may reduce HSV detection and viral load in those with HIV co-infection, their impact on HSV transmission needs to be confirmed. When prescribing suppressive antivirals for patients with HSV and HIV co-infection, clinicians should caution patients that suppressive therapy may not reduce risk of HSV transmission to susceptible partners.

\section{Acknowledgements}

The authors would like to thank Shalane Ha for her work as a third reviewer for eligibility screening and risk of bias assessment, and Portfolio Librarian Ella Westhaver for her assistance with the systematic search and retrieval of literature. The authors would also like to thank Margaret Gale-Rowe, Karen Timmerman, Jun Wu and Cathy Latham-Carmanico for their helpful comments on this review.

\section{Conflict of interest}

There are no conflicts of interest to declare.

\section{Funding}

No funds were received for this study.

\section{References}

1. Rotermann M, Langlois KA, Severini A, Totten S. Prevalence of Chlamydia trachomatis and herpes simplex virus type 2: results from the 2009 to 2011 Canadian Health Measures Survey. Health Rep. 2013;24(4):10-5.

2. Smith JS, Robinson NJ. Age-specific prevalence of infection with herpes simplex virus types 2 and 1: a global review. J Infect Dis. 2002;186 (Suppl 1):S3-28.

3. Freeman EE, Weiss HA, Glynn JR, Cross PL, Whitworth JA, Hayes RJ. Herpes simplex virus 2 infection increases HIV acquisition in men and women: systematic review and metaanalysis of longitudinal studies. AIDS. 2006;20:73-83.

4. Van de Perre $P$, Segondy $M$, Foulongne $V$, Ouedraogo A, Konate I, Huraux JM, et al. Herpes simplex virus and HIV-1: deciphering viral synergy. Lancet Infect Dis. 2008;8:490-7. 
5. Schacker T, Zeh J, Hu HL, Hill J, Corey L. Frequency of symptomatic and asymptomatic HSV-2 reactivations among HIV-infected men. J Infect Dis. 1998;178:1616-22.

6. Augenbraun M, Feldman J, Chirgwin K, Zenilman J, Clarke L, DeHovitz J, et al. Increased genital shedding of herpes simplex virus type 2 in HIV-seropositive women. Ann Intern Med. 1995;123:845-7.

7. Freeman EE, Weiss HA, Glynn JR, Cross PL, Whitworth JA, Hayes RJ. Herpes simplex virus 2 infection increases HIV acquisition in men and women: systematic review and metaanalysis of longitudinal studies. AIDS. 2006;20(1):73-83.

8. Mertz GJ. Asymptomatic shedding of herpes simplex virus 1 and 2: implications for prevention of transmission. J Infect Dis. 2008;198(8):1098-100.

9. Corey L, Wald A, Patel R, Sacks SL, Tyring SK, Warren T, et al. Valacyclovir HSV Transmission Study Group. Once-daily valacyclovir to reduce the risk of transmission of genital herpes. N Engl J Med. 2004;350(1):11-20.

10. Ruddock B, Severn M; Canadian Agency for Drugs and Technologies in Health. Health Technology Inquiry Service. Oral antivirals for the treatment and prevention of orolabial and genital herpes. The Health Technology Inquiry Service. Ottawa (ON): Canadian Agency for Drugs and Technology in Health; 2007.

11. Jungmann EM. Genital herpes. BMJ Clin Evid. 2007;2007:1603.

12. Higgins JP, Altman DG, Gøtzsche PC, Jüni $P$, Moher D, Oxman AD, et al. The Cochrane Collaboration's tool for assessing risk of bias in randomised trials. Br Med J. 2011;343:d5928.

13. Nijhawan AE, DeLong AK, Chapman S, Rana A, Kurpewski $\mathrm{J}$, Ingersoll J, et al. Effect of HSV-2 suppressive therapy on genital tract HIV-1 RNA shedding among women on HAART: a pilot randomized controlled trial. Infect Dis Obstet Gynecol. 2012;2012:868526.

14. Mujugira A, Magaret AS, Celum C, Baeten JM, Lingappa JR, Morrow RA, et al. Daily acyclovir to decrease herpes simplex virus type 2 (HSV-2) transmission from HSV-2/HIV-1 coinfected persons: a randomized controlled trial. J Infect Dis. 2013; 208(9):1366-74.

15. Baeten JM, Strick LB, Lucchetti A, Whittington WL, Sanchez J, Coombs RW, et al. Herpes simplex virus (HSV)-suppressive therapy decreases plasma and genital HIV-1 levels in HSV-2/ HIV-1 coinfected women: a randomized, placebo-controlled, cross-over trial. J Infect Dis. 2008;198(12):1804-8.

16. Cowan FM, Pascoe SJ, Barlow KL, Langhaug LF, Jaffar S, Hargrove JW, et al. A randomised placebo-controlled trial to explore the effect of suppressive therapy with acyclovir on genital shedding of HIV-1 and herpes simplex virus type 2 among Zimbabwean sex workers. Sex Transm Infect. 2008;84(7):548-53.

17. Delany S, Mlaba N, Clayton T, Akpomiemie G, Capovilla A, Legoff J, et al. Impact of aciclovir on genital and plasma HIV-1 RNA in HSV-2/HIV-1 co-infected women: a randomised placebo-controlled trial in South Africa. AIDS. 2009;23(4):461-9.

18. Dunne EF, Whitehead S, Sternberg M, Thepamnuay S, Leelawiwat W, McNicholl JM, et al. Suppressive acyclovir therapy reduces HIV cervicovaginal shedding in HIV- and HSV-2-infected women, Chiang Rai, Thailand. J Acq Immun Defic Syndr. 2008;49(1):77-83.

19. Kim HN, Wang J, Hughes J, Coombs R, Sanchez J, Reid S, et al. Effect of acyclovir on HIV-1 set point among herpes simplex virus type 2-seropositive persons during early HIV-1 infection. J Infect Dis. 2010;202(5):734-8.

20. Nagot N, Ouédraogo A, Foulongne $V$, Konaté I, Weiss $H A$, Vergne L, et al. Reduction of HIV-1 RNA levels with therapy to suppress herpes simplex virus. New Engl J Med. 2007;356(8):790-9.

21. Ouedraogo A, Nagot N, Vergne L, Konate I, Weiss $H A$, Defer MC, et al. Impact of suppressive herpes therapy on genital HIV-1 RNA among women taking antiretroviral therapy: a randomized controlled trial. AIDS. 2006;20(18):2305-13.

22. Perti T, Saracino M, Baeten JM, Johnston C, Diem K, Ocbamichael N, et al. High-dose valacyclovir decreases plasma HIV-1 RNA more than standard-dose acyclovir in persons coinfected with HIV-1 and HSV-2: a randomized crossover trial. J Acq Immun Def Synd. 2013;63(2):201-8.

23. Tanton C, Weiss HA, Rusizoka M, LeGoff J, Changalucha J, Baisley K, et al. Long-term impact of acyclovir suppressive therapy on genital and plasma HIV RNA in Tanzanian women: a randomized controlled trial. J Infect Dis. 2010;201(9):1285-97.

24. Tobian AA, Grabowski MK, Serwadda D, Newell K, Ssebbowa P, Franco V, et al. Reactivation of herpes simplex virus type 2 after initiation of antiretroviral therapy. J Infect Dis. 2013;208(5):839-46.

25. Van Wagoner N, Geisler WM, Bachmann LH, Hook EW. The effect of valacyclovir on HIV and HSV-2 in HIVinfected persons on antiretroviral therapy with previously unrecognised HSV-2. Int J STD AIDS. 2015;26(8):574-81.

26. Zuckerman RA, Lucchetti A, Whittington WL, Sanchez J, Coombs RW, Zuñiga R, et al. Herpes simplex virus (HSV) suppression with valacyclovir reduces rectal and blood plasma HIV-1 levels in HIV-1/HSV-2-seropositive men: a randomized, double-blind, placebo-controlled crossover trial. J Infect Dis. 2007;196(10):1500-8.

27. Reyes M, Shaik NS, Graber JM, Nisenbaum R, Wetherall NT, Fukuda K, et al. Acyclovir-resistant genital herpes among persons attending sexually transmitted disease and human immunodeficiency virus clinics. Arch Intern Med. 2003;163(1):76-80.

28. DeJesus E, Wald A, Warren T, Schacker TW, Trottier S, Shahmanesh $M$, et al. Valacyclovir for the suppression of recurrent genital herpes in human immunodeficiency virusinfected subjects. J Infect Dis. 2003;188(7):1009-16. 